Proceedings of the International School and Conference on Optics and Optical Materials, ISCOM07, Belgrade, Serbia, September 3-7, 2007

\title{
On Bright and Dark Breathers in Lattices with Saturable Nonlinearity
}

\author{
A. MAluckov ${ }^{a, *}$, M. Stepić ${ }^{b}$ And LJ. HAdŽIEvski ${ }^{b}$ \\ ${ }^{a}$ Faculty of Sciences and Math., University of Niš \\ P.O.B. 224, 18001 Niš, Serbia \\ ${ }^{b}$ Vinča Institute of Nuclear Sciences, P.O.B. 522, 11001 Belgrade, Serbia \\ The moving bright and dark localized modes in one-dimensional optical \\ lattices with saturable nonlinearity are considered with respect to the grand \\ canonical free energy concept and linear stability analysis of the eigenvalue \\ spectra. \\ PACS numbers: 63.20.Ry, 05.45.Yv, 42.65.Tg, 03.75.Lm
}

\section{Introduction}

In the past two decades the special attention is devoted to the strongly localized modes [1] which are promising candidates for guiding, steering, and switching of light beams in the nonlinear optical lattices. The effect of energy localization through discreteness and nonlinearity is universal and relevant for many non-optical systems, such as DNA-promoter dynamics [2], discrete breathers in a Josephson ladder [3], localized modes in the anharmonic crystals [4], or localization of matter waves in the Bose-Einstein condensates using optically-induced periodic potentials [5].

Bright discrete solitons have been studied in detail in photonic lattices exhibiting cubic, quadratic, saturable, and nonlocal nonlinearity $[1,6-9]$. Because of various experimental difficulties and demand for higher input power dark solitons are investigated discernible rarely when compared to their bright analog $[10,11]$. Very recently, stable propagation of on-site and inter-site dark solitons were experimentally observed in the self-defocusing lithium niobate waveguide arrays with saturable nonlinearity at microwatt power level $[12,13]$.

In this paper the comparative study of the moving localized bright and dark modes in lattices with saturable nonlinearity [14] is presented. This study is based on the grand canonical free energy concept [15] and analysis of the eigenvalue spectra of the localized configurations.

${ }^{*}$ corresponding author; e-mail: sandra@pmf.ni.ac.yu 


\section{Model equation}

The model equation is the one-dimensional discrete nonlinear Schrödinger equation with saturable nonlinearity [9]:

$$
\mathrm{i} \partial U_{n} / \partial t+U_{n+1}+U_{n-1}-2 U_{n}+\gamma U_{n} /\left(1+\left|U_{n}\right|^{2}\right)=0
$$

where $U_{n}$ is the normalized wave function in the $n$-th lattice element $(n=1,2 \ldots)$, and $\gamma$ is the nonlinearity parameter. For $\gamma>0$ the nonlinearity is defocusing (DF) and for $\gamma<0$ is self-focusing (SF).

Equation (1) represents a system of coupled nonlinear difference-differential equations which are not integrable in general case but possess two conserved quantities, $H=\sum_{n}\left[-\gamma \ln \left(1+\left|U_{n}\right|^{2}\right)+\left|U_{n-1}-U_{n}\right|^{2}\right]-$ Hamiltonian and $P=$ $\sum_{n}\left|U_{n}\right|^{2}$ - norm (power). The model equation supports localized solutions of various types [1]. The assumption $U_{n}(t)=\phi_{n} \mathrm{e}^{\mathrm{i} \omega t}$, where $\omega$ is the propagation parameter, leads Eq. (1) to the steady state equation

$$
-\omega \phi_{n}+\phi_{n+1}+\phi_{n-1}-2 \phi_{n}+\gamma \phi_{n} /\left(1+\phi_{n}^{2}\right)=0
$$

where for the cw solution the envelope can be taken in the form $\phi_{n}=U_{\mathrm{c}} \mathrm{e}^{\mathrm{i} K_{\mathrm{s}} n}$. The $K_{s}=\pi$ for staggered and $K_{s}=0$ for unstaggered solutions.

The stability of the stationary modes is studied by solving the eigenvalue problem of the small complex perturbations $\epsilon_{n}$ to the wave envelopes. After short and simple algebraic procedure the eigenvalue (EV) matrix can be obtained [13]. The EV matrix for the lattice with $N$ elements has dimension $2 N \times 2 N$. The linear stability analysis for the soliton solutions is based on the properties of the EVs of this matrix [13].

For the $\mathrm{cw}$ solution the dispersion relation is analytically obtained in [13]. From the condition for the modulation instability it was concluded: for DF nonlinearity the creation of stable staggered bright and unstaggered dark solitons, while for SF nonlinearity the creation of stable unstaggered bright and staggered dark solitons is possible. For all localized modes two different configurations can exist: the on-site, centered on the lattice element and inter-site, centered between two neighboring lattice elements [14].

The dynamical properties of the unstaggered bright localized modes in the lattices with saturable nonlinearity are determined by the cascade amplitude saturation mechanism [9]. The main consequences are alternation of the stability between the on-site and inter-site configurations for fixed value of $P$, and the existence of the moving bright modes for a few high values of power [14]. On the other hand, the phase flip between the neighboring lattice grids changes the properties of the bright staggered modes in comparison with the unstaggered bright modes and above mentioned stability alternation disappears.

The particularity of the dark solitons is that they exist on a stable background wave [10]. Therefore, the analysis of the dark localized mode is not possible without inclusion of the background oscillations.

Grand canonical free energy. To describe the localized mode behaviour, the 
grand canonical free energy concept is developed [15]. The system - localized mode and background - is characterized by the grand canonical free energy $G$ which is defined as $G=H-\omega P$ for bright (Fig. 1b,c) and $G=H_{\mathrm{c}}-\omega P_{\mathrm{c}}$ for dark modes (Fig. 2c). The conserved complementary norm $P_{\mathrm{c}}$ and complementary Hamiltonian $H_{\mathrm{c}}$ are introduced for the localized dark configurations because the conserved quantities $P$ and $H$ in these cases diverge [10,13], $P_{\mathrm{c}}=N U_{\mathrm{cw}}^{2}-P, H_{\mathrm{c}}=$ $H+\gamma\left(P_{\mathrm{c}} /\left(1+U_{\mathrm{cw}}^{2}\right)-N \ln \left(1+U_{\mathrm{cw}}\right)\right)$. The $U_{\mathrm{cw}}$ is the (Bloch-wave) background.

For one particular unstaggered localized solution characterized by the value of $P$, or $P_{\mathrm{c}}$, the stable configuration between the on-site and inter-site configuration is this one which is associated with the smaller value of $G$, Figs. 1b,c, 2c - the grand canonical free energy minima criteria. In the case of staggered localized configurations according to the previous studies [1] the criterion for stability is opposite.

\section{Bifurcation analysis}

\subsection{Bright localized modes}

In the case of the bright unstaggered localized modes only pure real and pure imaginary EVs over the whole existence region are found which indicates the possibility of the exponentially growing instability.

Considering the EV spectra, increasing the value of parameter $P$ for fixed $\gamma$ and $\omega$, the merging of two real EV branches after which only the imaginary EVs sustain for on-site mode is followed by the creation of two purely real EVs from the purely imaginary EVs spectrum for inter-site mode, and vice versa. This behavior can be associated with tangential (saddle-center) [16] bifurcation for both the on-site and inter-site localized modes. Only in several intervals of $P$ both the on-site and inter-site configurations are simultaneously characterized by the pure imaginary EV spectrum, Fig. 1a, where both configurations are neutrally stable.
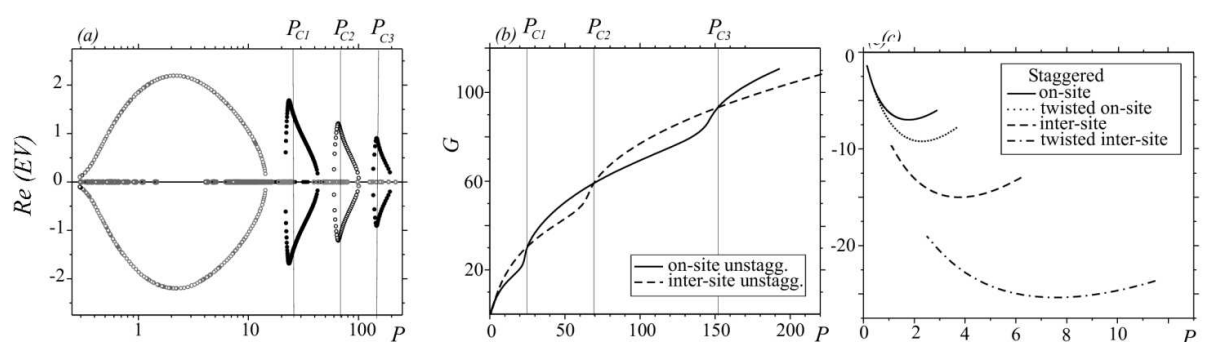

Fig. 1. The EV spectra for the on-site (black) and the inter-site unstaggered bright localized mode (gray circles) (a). The $G(P)$ diagrams are shown for unstaggered (b) and for staggered case (c). The $P_{C i}, i=1,2,3$ are transparent points.

The previously mentioned properties of the EV spectrum can be related to the alternation of the mode stability, i.e. the stable on-site and unstable inter-site mode are followed by unstable on-site and stable inter-site mode, and vice versa. 
In Fig. 1a,b the transparent points [14] are shown. They coincide with the points of simultaneously neutral stability of the on- or inter-site localized configuration and maximal instability growth rate of the corresponding inter- or on-site configuration, respectively. Between two mentioned configurations neutrally stable one can be transversely moved.

The EV spectra of the staggered bright configurations are formed of the purely real and purely imaginary EVs, as in the unstaggered cases. Numerically it is confirmed that both on- and inter-site configurations are unstable in the most part of the existence region [17]. On the other hand, the EV spectrum of twisted ("composite") staggered localized modes [1], in addition to the pure imaginary EVs has the complex EVs for small $P$ and pure real EVs for higher $P$. This indicates the possibility for oscillatory and exponential instability, respectively.

The stability alternation between on-site and inter-site configurations is absent for staggered bright localized modes [9]. In addition, the bright staggered modes are very inert to the transverse kicks which can be associated with clear separation (high energy barrier) between the $G(P)$ curves for on-site and inter-site staggered configurations [8].

\subsection{Dark localized modes}

The EV spectrum of dark localized modes has contributions from [13] continuous part (from background) and discrete part (from localized structure). The discrete EV spectrum by itself and through the interaction with the continuous part of the EV spectrum can be associated with the eventual instability of the solitons. Generally, for both the on-site and inter-site staggered and unstaggered dark configurations a subset with pure imaginary EVs inside the continuous part of the EV spectrum (Fig. 2) is observed, which is well described by the dispersion curve [13].


Fig. 2. EV spectrum for the on-site (a) and the inter-site dark unstaggered solitons. Dashed black - pure imaginary EVs (only the extremal EVs are plotted), white line the real and black line - the imaginary part of complex EVs, black line in (b) - pure real EVs. The continuous spectrum is displayed as a shaded region. Index $b$ denotes bifurcation values. The $G_{\mathrm{c}}\left(P_{\mathrm{c}}\right)$ dependence is plotted in (c).

For the on-site dark configuration the four branches with complex eigenvalues of the discrete EV spectrum coexist with the $2 N-4$ purely imaginary EVs in 
some intervals of the system parameters, Fig. 2a. This indicates presence of the oscillatory instability. On the other hand, for the inter-site dark configuration numerical results show existence of two branches with pure real EVs and $2 \mathrm{~N}-2$ purely imaginary EVs in some regions of parameters, which indicates presence of the exponentially growing instability (Fig. 2b). Fixing $\omega$ and $\gamma$ and increasing the value of parameter $P_{\mathrm{c}}$ for on-site dark configuration the bifurcation of the Hopf type [16] and for inter-site configuration tangential bifurcation [16] can be identified. The bifurcation points coincide with the intersection of the discrete and continuous part of the EV spectrum as shown in Figs. 2a and b.

All configurations of the dark solitons are unstable in the existence region except in parts with only purely imaginary EVs. There the existence of stable dark breathers is confirmed [13]. These results are consistent with the recently published experimental results [12].

\section{Transverse motion}

The difference between the grand canonical free energy of the on-site and inter-site localized configurations with the same norm $P$ for bright, and $P_{\mathrm{c}}$ for dark modes has a sense of the potential barrier which arises from the discreteness of the system. It can be associated with a measure of the well-known Peierls-Nabarro $(\mathrm{PN})$ barrier.

The grand canonical free energy for the bright and dark soliton configurations is displayed in Figs. 1b,c, 2c. The remarkable feature of stability alternation between on-site and inter-site configurations observed for the unstaggered bright solitons in lattices with SF nolinearity is absent for dark localized modes [14]. There are no discrete transparent points which correspond to the zeros of the $\Delta G$. Let us note that the moving bright breather can exist only in the transparent points [14]. Instead, for dark modes, as can be clearly seen, the energy difference
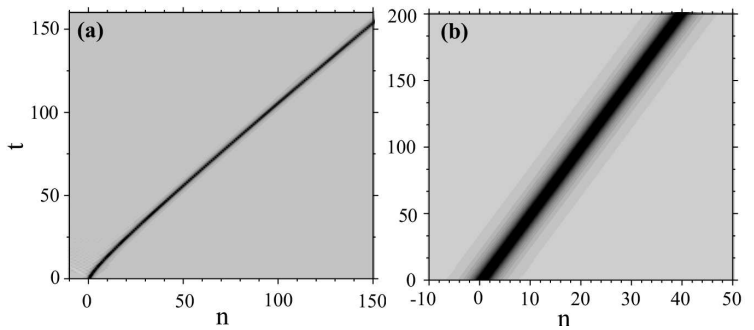

Fig. 3. The free transverse motion of the discrete breathers: moving dark breather for $\omega=0.08$ (a) and for $\omega=8.9(\mathrm{~b})$.

$\Delta G\left(P_{\mathrm{c}}\right)$ for $P_{\mathrm{c}} \ll 1$ and $P_{\mathrm{c}} \gg 1$ vanishes which coincides with the regions of the existence of the stable dark breathers [13]. There the dark breathers are not affected by the PN barrier and can freely move across the lattice elements, Fig. 3. 


\section{Conclusion}

In conclusion, the existence of moving bright and dark breathers in lattices with saturable nonlinearity is confirmed by stability analysis. The existence regions coincide with the zeros of the corresponding grand canonical free energy. In these regions in the absence of transverse perturbation stable propagation of the localized breathers is shown. This is consistent with the experimental observations.

\section{References}

[1] Yu.S. Kivshar, G.P. Agrawal, Optical Solitons: from Fibers to Photonic Crystals, Academic Press, San Diego 2003.

[2] M. Salerno, Phys. Rev. A 44, 5292 (2001).

[3] P. Binder, D. Abraimov, A.V. Ustinov, Phys. Rev. E 62, 2858 (1999).

[4] A.J. Sievers, S. Takeno, Phys. Rev. Lett. 61, 970 (1988).

[5] A. Trombettoni, A. Smerzi, Phys. Rev. Lett. 86, 2353 (2001).

[6] A.B. Aceves, G.G. Luther, C. De Angelis, A.M. Rubenchik, S.K. Turisyn, Phys. Rev. Lett. 75, 73 (1995).

[7] H.S. Eisenberg, Y. Silberberg, R. Morandotti, A.R. Boyd, J.S. Aitchison, Phys. Rev. Lett. 81, 3383 (1998).

[8] N.E. Efremidis, S. Sears, D.N. Christodoulides, J.W. Fleischer, M. Segev, Phys. Rev. E 66, 046602 (2002).

[9] M. Stepić, D. Kip, Lj. Hadžievski, A. Maluckov, Phys. Rev. E 69, 066618 (2004).

[10] Yu.S. Kivshar, W. Królikowski, O. A. Chubykalo, Phys. Rev. B 50, 5020 (1994).

[11] R. Morandotti, H.S. Eisenberg, Y. Silnerberg, M. Sorel, J.S. Aitchison, Phys. Rev. Lett. 86, 3296 (2001).

[12] E. Smirnov, C.E. Rüter, M. Stepić, D. Kip, V. Shandarov, Phys. Rev. E 74, 065601(R) (2006).

[13] Lj. Hadžievski, A. Maluckov, M. Stepić, Opt. Express 15, 5687 (2007).

[14] Lj. Hadžievski et al, Phys. Rev. Lett. 93, 033901 (2004).

[15] T.R.O. Melvin, A.R. Champneys, P.G. Kevrekidis, J. Cuevas, Phys. Rev. Lett. 97, 124101 (2006).

[16] S. Wiggins, Global Bifurcations and Chaos: Analytical Methods, Springer-Verlag, New York 1988.

[17] E.P. Fitrakis, P.G. Kevrekidis, H. Susanto, D.J. Frantzeskakis, Phys. Rev. E 75, 066608 (2007). 